\title{
Practice Schedule and Cognitive Style Interaction in Learning a Maze Task
}

\author{
OTTO JELSMA and JULES M. PIETERS \\ Department of Education, University of Twente, The Netherlands
}

\begin{abstract}
SUMMARY
In the present study the effects of contextual interference on the retention and transfer performance of reflectives and impulsives on a maze task were studied. Forty-seven subjects were randomly assigned to either a high contextual interference group or to a low contextual interference group. Within the two groups subjects were further classified according to their preferred modes of responding. Retention and transfer were measured immediately following practice and after a 4-week delay. The dependent variables were tracing time and errors. Reflectives made fewer errors and moved more quickly after practising under conditions of high contextual interference. Impulsives tended to have fewer errors after practising under conditions of high contextual interference but moved more slowly. Based on these results it was suggested that trainers consider individual differences in reflectivityimpulsivity before designing particular practice schedules.
\end{abstract}

Almost any skill training programme emphasizes retention of skills that were practised during acquisition and in addition their transfer to a variety of different but related task demands in various situations (Annett and Piech, 1985; Annett and Sparrow, 1985). Recent explorations of contextual interference in motor skill learning suggest that retention and transfer are enhanced if the training programme forces subjects to increase the extent of cognitive processing during skill acquisition (Battig, 1979; Shea and Morgan, 1979; Del Rey, Wughalter and Whitehurst, 1982; Shea and Zimny, 1983; Lee and Magill, 1983; Lee, Magill and Weeks, 1985; Lee and Magill, 1985). Contextual interference refers to interference of cognitive operations underlying goal-directed actions. Interference can be brought about by changing task demands across acquisition trials (Battig, 1979). Interference is high if task demands vary from trial to trial, which is the case if several task variants are practised in a random order (hereafter denoted as 'random practice schedule'); interference is low if task demands are held relatively constant across acquisition trials, which is realized if task variants are practised in blocks of trials (hereafter denoted as 'blocked practice schedule').

As results of many studies show, interference may be detrimental to performance during acquisition but produces a facilitation in retention and transfer. For instance, using a tri-component, rapid movement task, Shea and Morgan (1979) had subjects learn three response patterns under either a random practice schedule or a blocked practice schedule. Retention of the practised response patterns and transfer to another response pattern were measured immediately following training and after a 10-day delay. With reaction time and movement time as dependent 
variables, results showed lower performances during acquisition, but superior performances on retention tests and transfer tests in the random practice schedule. To explain the advantage of a random practice schedule over a blocked practice schedule Shea and Zimny (1983) suggest that experience with intervening, related activities during practice may lead to greater elaboration (encoding of additional information) and hence to better learning. On the other hand, though not refuting the potential benefits of elaboration, Lee and Magill (1985) suggest that a greater cognitive effort during practice as a result of regeneration of action plans explains the observed results. Both explanations demonstrate the dominant role of conscious mechanisms that subserve the translation from intention-to-action in skill learning (Schneider and Shiffrin, 1977; Shiffrin and Schneider, 1977; Fisk and Schneider, 1984).

According to Shea and Morgan (1979) the findings of studies on contextual interference can be readily applied to an instructional setting. They note that in most skill training programmes one task per session is practised in order to avoid confusing the learner, presumably giving the learner the opportunity to learn the task completely before attempting to learn a similar one. Shea and Morgan (1979) suggest that, instead, a number of tasks during each session for a number of sessions should be trained in order to achieve maximum retention and transfer. However, before recommending the application of contextual interference in instructional settings, it should be examined if the ability to learn and perform well under certain practice conditions may be associated with individual differences (e.g. Ackerman and Schneider, 1984). For instance, up till now studies on contextual interference did not control for subjects' cognitive style. Cognitive style refers to individual variations in information processing and response generation. Research has shown that learners prefer to work and succeed to a greater extent when using their dominant cognitive style (Lyons, 1984; Lyons and Languis, 1985). Consequently, an optimal level of achievement is thought to be dependent upon the ideal matching of a person's cognitive style and instructional conditions (Cronbach and Snow, 1977; Singer and Gerson, 1979).

Out of many cognitive styles that have been identified, reflectivity-impulsivity (Kagan, Rosman, Day, Albert and Phillips, 1964) may be of particular interest to those who design training programmes. Reflectivity refers to a speed-accuracy trade-off in the direction of accuracy, whereas impulsivity is associated with responding quickly and not taking the time to carefully select the correct solution. In general, when several possible alternatives are available and there is some uncertainty over which one is the most appropriate, reflectives make less errors because they reflect more on response alternatives, show a more efficient deployment of attention and make more efficient use of feedback information than impulsives (see for a review Messer, 1976).

The purpose of the present study was to determine the effects of contextual interference on the retention and transfer performance of reflectives and impulsives. Four variants of a maze task were practised either in a random practice schedule or in a blocked practice schedule. It is hypothesized that on retention tests and transfer tests reflectives who practised in a learning situation that calls heavily on reflection - as under high contextual interference conditions-will move more quickly and will make fewer errors as compared to reflectives who practised in a well-structured learning situation not strongly calling on reflection-as under low 
contextual interference conditions. Impulsives who practised in the random practice schedule are expected to move more slowly and they are expected to make more errors than impulsives who practised in the blocked practice schedule.

\section{METHOD}

\section{Subjects}

Forty-seven right-handed university students (nine males and 38 females; mean age $=20.3$ years, $\mathrm{SD}=1.4$ years) participated in the experiment for course credit.

Statistical analyses were performed only on performances of subjects with either a clear-cut reflective or impulsive cognitive style as identified by the Matching Familiar Figures Test (MFFT) (Kagan et al., 1964). The test consisted of 12 standard pictures each having six alternatives. A visual match to standard was required. Subjects who scored above the median in total response time and below the median in total number of errors were classified as reflective; subjects who scored below the median in total response time and above the median in total number of errors were identified as impulsive. Based on this procedure the scores for 14 subjects did not fall into these distinct categories and their performances were not included in further analyses. This reduced the number of subjects in the random practice schedule to 16 (10 reflectives and six impulsives) and the number of subjects in the blocked practice schedule to 17 (eight reflectives and nine impulsives).

\section{Apparatus}

The experiment was conducted in a sound-proof cubicle. Subjects were seated at a table in a comfortable chair. A screen was placed at eye level in front of the subject. Within easy reach a normal joystick, located opposite the midline of the subject's body, and a separate starting button, located to the right of the subject's body, were mounted on the table. Auditory feedback was realized as follows: whenever the subject moved the joystick in a wrong direction (error), an auditory signal was channelled by a normal audio amplifier and fed back to the subject via a loudspeaker. The experiment was controlled by a PDP-11/03 minicomputer. Errors, planning time, and tracing time were recorded automatically and stored on floppy discs for later analyses. 'Planning time' was operationalized as time between the onset of a starting signal and the initiation of the first cursor displacement. 'Tracing time' was operationalized as time between the first cursor displacement and reaching the finish.

\section{Task}

Subjects had to trace at their own pace, and with minimal errors, four variants (rotations and mirror-images) of a complex geometrical pattern, called 'meander', with a joystick-controlled cursor (Fig. 1). The variants were hidden in a maze.

Each variant was defined by a unique starting point and finish as indicated by the cursor (displayed as an asterisk) and an opening in the surrounding framework of 
A
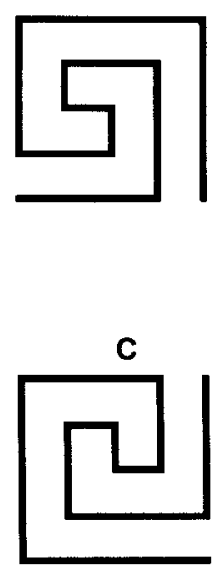

B

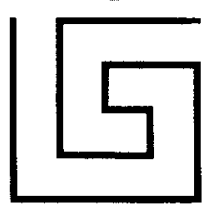

D

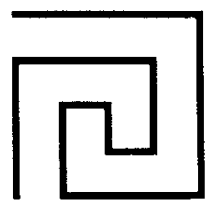

Figure 1. Four variants of the meander $\left(\mathrm{C}\right.$ and $\mathrm{D}$ are horizontally reflected and rotated $90^{\circ}$ with respect to $A$ and $B ; B$ and $D$ are rotated $180^{\circ}$ with respect to $A$ and $C$ )

the maze, respectively. The maze was displayed on the screen in a matrix of $19 \times 19$ characters (Figs $2 \mathrm{~A}$ and $2 \mathrm{~B}$ ).

Besides visible barriers (the black squares and the surrounding framework) there were also barriers (the shaded area in Fig. 2A) that were not visible for the subjects during task performance. These invisible barriers could be transposed so that always only one variant fitted into the maze. In this way there was always only one correct pathway at a time through the maze.

A

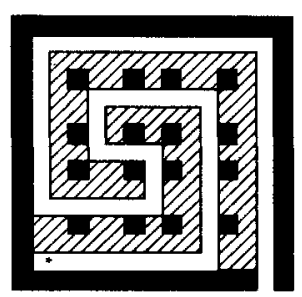

B

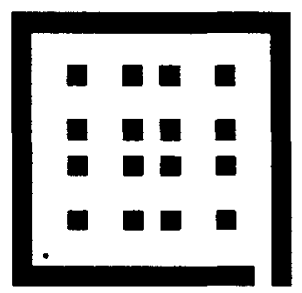

Figure 2. The maze with all barriers (visible and invisible) shown (A) and the maze as it was displayed for subjects on the screen $(B)$

\section{Procedure}

The experiment was divided into two sessions. The first session consisted of an acquisition phase, a retention test and a transfer test. The MFFT was administered during the first session. The second session, conducted after a 4-week delay, consisted of a retention test and a transfer test only. At the beginning of each session subjects were given standardized instructions to explain the purpose of the task. It was stressed that they had to reduce errors and that they could perform the task self-paced. It was furhter explained that the experiment was completely 
computer-controlled. Subjects were not informed of the fact that, in addition to errors, tracing time was also used as a measure of performance. Due to this, it was expected that this latter variable would produce the largest differences between reflectives and impulsives. The acquisition phase started by a subject's push on the starting button. Acquisition phase, retention test and transfer test are described separately.

\section{Acquisition}

The nine males and 38 females were randomly assigned to either a random practice schedule $(n=23)$ or a blocked practice schedule $(n=24)$. Each subject received 40 acquisition trials-10 trials of every variant. Subjects in the blocked practice schedule always completed all trials on a variant before the next was introduced. The order in which variants were presented was counterbalanced across subjects. Subjects in the random practice schedule were given acquisition trials in a random order. No trials of the same variant occurred consecutively.

Prior to the start of the acquisition phase, subjects were given 1 minute's practice with the control of the joystick. Then subjects were requested to start the acquisition phase by pushing the starting button. Prior to each trial, an example of the variant at hand was shown. The example consisted of a correct passage of the cursor through the maze at the speed of 15 characters per second. Two seconds after the example was shown an auditory signal indicated the beginning of a trial. Whenever an error was made the cursor stopped and auditory feedback was given. A trial was completed as soon as the cursor reached the finish.

After finishing a trial subjects obtained knowledge of results (KR), consisting of the number of errors they made. Planning time and tracing time were not shown. KR was displayed 4 seconds on the screen. Then again subjects were requested to push the starting button for the next example and trial. The acquisition phase ended as soon as the subject had performed 40 trials. Following the acquisition phase a 5-minute rest was provided.

After the break the MFFT was administered by using the items and instructions originally developed by Kagan et al. (1964). The experimenter recorded time to first response and number of errors per item. Afterwards, total time to first response and total number of errors were determined.

\section{Retention}

Following MFFT both acquisition groups received 16 retention trials (four of every variant). Retention trials were identical to acquisition trials, except the presentation of an example. Retention trials were presented in a counterbalanced order. Subjects were assumed to recognize each variant by its starting point and finish.

Following the first retention test, subjects were given a 2-minute rest, after which the first transfer test was administered. After a 4-week delay a second retention test identical to the first one, and a second transfer test, were administered.

\section{Transfer}

Four transfer trials were assigned to both acquisition groups. At the first transfer test (during the first session), subjects traced a fifth variant and at the second transfer test (during the second session) they traced a sixth variant. The presentation of the transfer trials was identical to the presentation of the acquisition 
trials. The new variants were defined by a particular starting point and finish. Moreover, an example of the correct pathway was shown prior to each transfer trial. Transfer trials were presented in a blocked order.

\section{Analysis}

Statistical analyses were performed separately on errors and tracing times. In all statistical analyses planning time was included as covariate. A three-way analysis of covariance over condition (random practice schedule vs. blocked practice shedule), cognitive style (reflective vs. impulsive) and trials (1 to 10) was conducted to test for significance of acquisition effects.

A four-way analysis of covariance over condition (random practice schedule vs. blocked practice schedule), cognitive style (reflective vs. impulsive), retention interval (immediately following training vs. 4-week delay) and trials ( 1 to 4) was conducted to test for significance of retention effects.

A second four-way analysis of covariance over condition (random practice schedule vs. blocked practice schedule), cognitive style (reflective vs. impulsive), transfer interval (immediately following training vs. 4-week delay) and trials (1 to 4) was conducted to test for significance of transfer effects.

\section{RESULTS}

Figures 3, 4 and 5 present the mean number of errors (A) and the mean tracing times (B) for the comparisons between the random practice schedule and the blocked practice schedule for reflectives and impulsives for acquisition, retention, and transfer. All presented means are adjusted for planning time.

\section{Acquisition data}

As is shown in Fig. 3A, reflectives made more errors in the blocked practice schedule than in the random practice schedule (15.4 vs. 8.1); impulsives seem to do about equally well in both practice groups (10.9 vs. 9.1$)$. The analysis of covariance indicated a significant condition $\times$ cognitive style interaction, $F(1,1279)=8.09, p<$ .01 . The mean number of errors in the random practice schedule was 8.6 and in the blocked practice schedule 13.2. This difference reached statistical significance, $F(1,1279)=22.75, p<.001$.

From Fig. $3 \mathrm{~B}$ it can be seen that reflectives moved more quickly in the random practice schedule than in the blocked practice schedule $(17.4 \mathrm{~s}$ vs. $19.9 \mathrm{~s})$; impulsives were faster in the blocked practice schedule than in the random practice schedule $(22.2 \mathrm{~s}$ vs. $24.3 \mathrm{~s})$. The interaction reached statistical significance, $F(1,1279)=9.89, p<.01$. Reflectives moved more quickly than impulsives in both practice group. The mean tracing time for reflectives was $18.6 \mathrm{~s}$ and for impulsives 23.2 s. The analysis of covariance indicated a significant cognitive style effect, $F(1,1279)=37.76, p<.001$. 


\section{ACQUISITION}

(A)

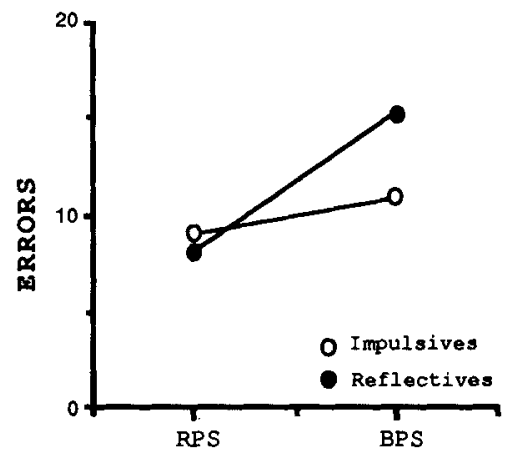

(B)

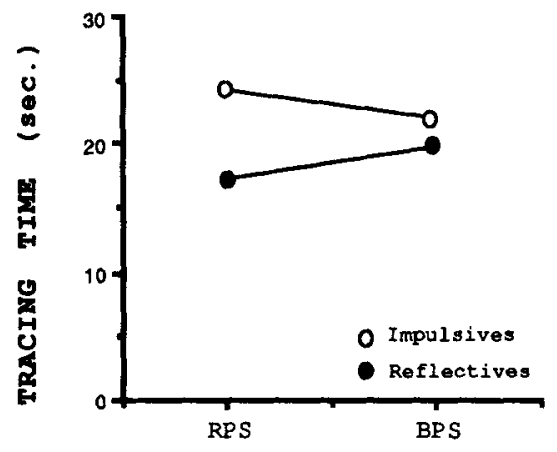

\section{PRACTICE SCHEDULE}

Figure 3. Mean number of errors (A) and mean tracing times (B) on acquisition for reflectives and impulsives in the random practice schedule (RPS) and the blocked practice schedule (BPS)

\section{Retention data}

Figure $4 \mathrm{~A}$ shows that reflectives and impulsives made about the same number of errors after practice in both the blocked practice schedule and the random practice schedule ( 6.0 and 3.2 vs. 5.6 and 3.5 , respectively). In the blocked practice schedule more errors were made than in the random practice schedule (5.8 vs. 3.3 ). The analysis of covariance indicated a significant Condition effect $F(1,1023)=$ $29.21, p<.001$.

Figure $4 \mathrm{~B}$ shows that reflectives in the random practice schedule moved more

\section{RETENTION}

(A)

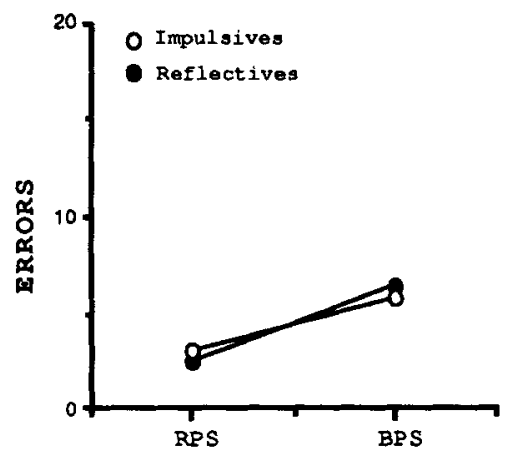

(B)

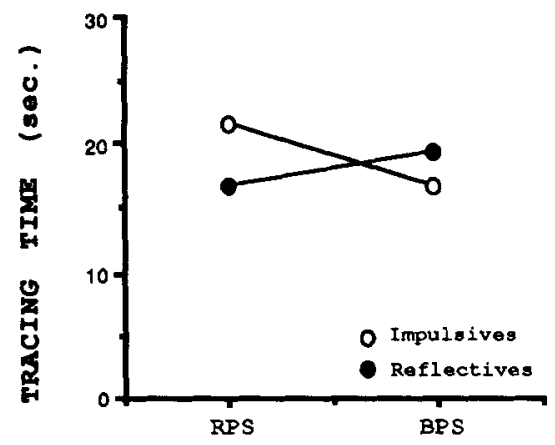

PRACTICE SCAEDULE

Figure 4. Mean number of errors (A) and mean tracing times (B) on retention for reflectives and impulsives in the random practice schedule (RPS) and the blocked practice schedule (BPS) 
quickly as compared to reflectives in the blocked practice schedule (17.6 s vs. 20.3 $\mathrm{s})$; impulsives were faster in the blocked practice schedule than in the random practice schedule $(17.5 \mathrm{~s}$ vs. $22.8 \mathrm{~s})$. The interaction reached statistical significance, $F(1,1023)=37.29, p<.001$.

\section{Transfer data}

From Fig. 5A it can be seen that for reflectives and impulsives the error data are parallel over the random practice schedule and the blocked practice schedule $(2.6$ vs. 4.8 and 4.4 vs. 7.3 , respectively). The mean number of errors for reflectives was 3.7 and for impulsives 5.9. The analysis of covariance indicated a significant cognitive style effect, $F(1,231)=12.22, p<.01$.

Figure $5 \mathrm{~B}$ shows that reflectives moved more quickly in the random practice schedule than in the blocked practice schedule (16.00 s vs. $20.2 \mathrm{~s})$; impulsives were faster in the blocked practice schedule than in the random practice schedule $(17.8 \mathrm{~s}$ vs. $22.4 \mathrm{~s})$. The interaction reached statistical significance, $F(1,231)=10.86$, $p<.01$.

\section{TRANSFER}

(A)

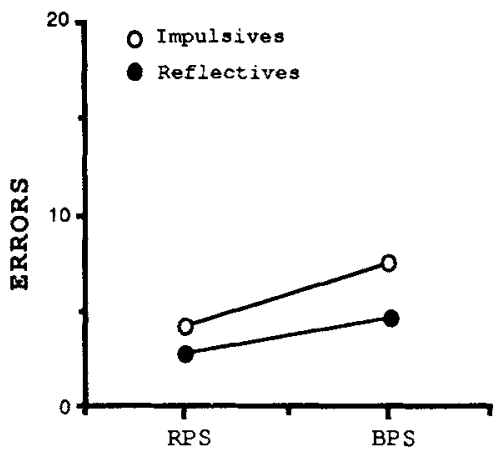

(B)

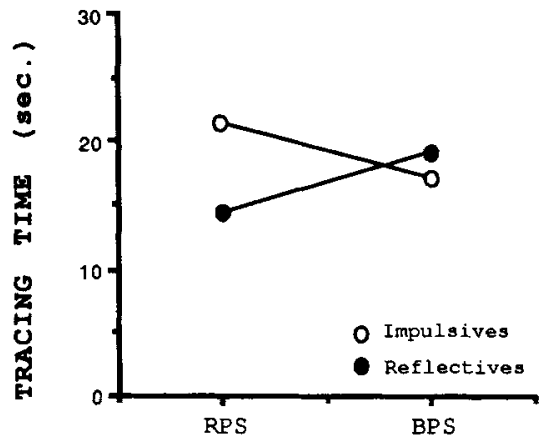

PRACTICE SCHEDULE

Figure 5. Mean number of errors (A) and mean tracing times (B) on transfer for reflectives and impulsives in the random practice schedule (RPS) and the blocked practice schedule (BPS)

\section{DISCUSSION}

The results of our study clearly demonstrate differential effects of contextual interference on performance of reflectives and impulsives. Acquisition data show that reflectives make more errors and move more slowly in a blocked practice shedule than in a random practice schedule. In the blocked practice schedule, reflectives make even more errors than impulsives, despite instructions to minimize 
error. Thus, for reflectives, the results support our hypothesis that the performance level decreases if the learning situation does not ideally match the preferred mode of responding. However, this cannot be concluded for impulsives. They seem to do equally well in both practice schedules although they take a relatively long time. In fact, reflectives move more quickly than impulsives in both practice schedules. This may seem surprising, recalling the fact that in novel situations reflectives usually perform slower and with greater accuracy than impulsives. However, possibly the particular instructions for task performance can account for this result. Subjects were explicitly instructed to make as few errors as possible. It was stressed that time was not used as a measure of performance. Although research has shown that forcing impulsives to slow down when solving problems did not automatically make them more accurate (e.g. Kagan, Pearson and Welch, 1966), our results seem to indicate that the reverse may be true: apparently instructions to minimize error can make impulsives adjust their speed-accuracy trade-off. This finding is consistent with research showing that impulsive behaviour can be modified with a training programme that emphasizes accuracy and de-emphasizes speed of response (Thompson, Teare and Elliott, 1983).

Only for reflectives, both retention and transfer data show the typical contextual interference effect in that they make fewer errors and move more quickly after practice in the random practice schedule, but make more errors and move more slowly after practice in the blocked schedule. In addition, on transfer tests reflectives make fewer errors than impulsives in both conditions. Because on retention tests no differences on errors were found between reflectives and impulsives, transfer data possibly reflect the more efficient way of information processing of reflectives during skill acquisition. Impulsives show a different pattern of speed-accuracy trade-off than reflectives. They tend to have more errors but move more quickly after practice in the blocked practice schedule, and they make fewer errors but move more slowly after practice in the random practice schedule. Thus, impulsives do not show the typical contextual interference effect. In addition, because it cannot be concluded that impulsives reached a higher performance level during and after practice in the blocked practice schedule, our findings are only partly consistent with results of research on cognitive styles that learners, when given the opportunity, succeed to a greater extent when using their dominant cognitive style. These results may be of particular interest to aptitude treatment interaction research (Cronbach and Snow, 1977).

As the acquisition data already indicated, impulsives seem to be willing to adjust their speed-accuracy trade-off in the direction of accuracy if they are instructed to do so. Our retention and transfer data show that this shift in the direction of what could be called reflective behaviour especially holds after practice under conditions that call on reflection. Thus, both task instructions and a particular organization of practice seem to be necessary if a shift in the direction of reflectivity is desired. Because impulsive behaviour is generally viewed as counterproductive to learning (e.g. Messer, 1976), these results may be of potential interest to research on intervention strategies to change an impulsive response style into a more reflective response style.

Based on our results it is concluded that the suggestion of Shea and Morgan (1979) that a number of tasks should be practised during each session for a number of sessions in order to achieve maximum retention and transfer cannot be 
generalized to all individuals. Before contextual interference can be successfully applied in instructional settings trainers should have to know if the learner tends to a reflective or to an impulsive cognitive style. Therefore, it is suggested that trainers consider individual differences in the reflective-impulsive dimension before designing a particular practice schedule.

\section{ACKNOWLEDGEMENTS}

We thank Sanne Dijkstra, Jeroen J. G. van Merriënboer, and an anonymous reviewer for their useful comments on earlier drafts of this article. We also thank Ben Reimerink for implementing the software for the experimental task. Requests for reprints should be sent to Otto Jelsma, Department of Education, University of Twente, POB 217, 7500 AE Enschede, The Netherlands.

\section{REFERENCES}

Ackerman, P. L. and Schneider, W. (1984). Individual differences in automatic and controlled information processing. Champaign, IL: University of Illinois, Human Attention Research Laboratory (Tech. Rep. No. HARL-ONR-8401).

Annett, J. and Piech, J. (1985). The retention of a skill following distributed training. Programmed Learning and Educational Technology, 22(2), 182-185.

Annett, J. and Sparrow, J. (1985). Transfer of training: a review of research and practical implications. Programmed Learning and Educational Technology, 22(2), 116-124.

Battig, W. F. (1979). The flexibility of human memory. In L. S. Cermak and F. I. M. Craik (Eds), Levels of processing and human memory. Hillsdale, $\mathrm{NJ}$ : Lawrence Erlbaum Associates, pp. 23-44.

Brown, H. J., Singer, R. N., Cauraugh, J. H. and Lucariello, G. (1985). Cognitive style and learner strategy interaction in the performance of primary and related maze tasks. Research Quarterly for Exercise and Sport, 56(1), 10-14.

Cronbach, L. J. and Snow, R. E. (1977). Aptitudes and instructional methods: a handbook for research on interactions. New York: Irvington.

Del Rey, P., Wughalter, E. H. and Whitehurst, M. (1982). The effects of contextual interference of females with experience in open sport skills. Research Quarterly for Exercise and Sport, 53(2), 108-115.

Fisk, A. D. and Schneider, W. (1984). Memory as a function of attention, level of processing, and automation. Journal of Experimental Psychology: Learning, Memory, and Cognition, 19, 181-197.

Kagan, J., Pearson, L. and Welch, L. (1966). Modifiability of an impulsive tempo. Journal of Educational Psychology, 57, 359-365.

Kagan, J., Rosman, B., Day, D., Albert, J. and Phillips, W. (1964). Information processing in the child: significance of analytic and reflective attitudes. Psychological Monographs, 78(1, Whole No. 578$)$.

Lee, T. D. and Magill, R. A. (1983). The locus of contextual interference in motor skill acquisition. Journal of Experimental Psychology: Learning, Memory and Cognition, 9(4), 730-746.

Lee, T. D. and Magill, R. A. (1985). Can forgetting facilitate skill acquisition? In D. Goodman, R. B. Wilberg and I. M. Franks (Eds), Differing perspectives in motor learning, memory and control. North-Holland: Elsevier, pp. 3-23.

Lee, T. D., Magill, R. A. and Weeks, D. J. (1985). Influence of practice schedule on testing schema theory predictions in adults. Journal of Motor Behavior, 17(3), 283-299.

Lyons, C. A. (1984). The relationship between prospective teachers' learning preference/style and teaching preference/style. Columbus: Ohio State University, 1984. 
Lyons, C. A. and Languis, M. L. (1985). Cognitive science and teacher education. Theory into Practice, 24(2), 127-130.

Messer, S. B. (1976). Reflection-impulsivity: a review. Psychological Bulletin, 83(6), 10261052.

Schneider, W. and Shiffrin, R. M. (1977). Controlled and automatic human information processing: I. Detection, search and attention. Psychological Review, 84, 1-66.

Shea, J. B. and Morgan, R. L. (1979). Contextual interference effects on the acquisition, retention and transfer of a motor skill. Journal of Experimental Psychology: Human Learning and Memory, 5(2), 9-187.

Shea, J. B. and Zimny, S. T. (1983). Context effects in memory and learning movement information. In R. A. Magill (Ed.), Memory and control of action. Amsterdam: NorthHolland, pp. 345-366.

Shiffrin, R. M. and Schneider, W. (1977). Controlled and automatic human information processing: II. Perceptual learning, automatic attending, and a general theory. Psychological Review, 84, 127-190.

Singer, R. N. and Gerson, R. F. (1979). Strategies, cognitive processes, and motor learning. In H. F. O'Neil, Jr and C. D. Spielberger (Eds), Cognitive and affective learning strategies. New York: Academic Press, pp. 215-247.

Thompson, R. W., Teare, J. F. and Elliott, S. N. (1983). Impulsivity: from theoretical constructs to applied interventions. Journal of Special Education, 17(2), 157-169. 